\section{Dritt- und Viertlinie beim NSCLC: Afatinib auch nach Progress noch nützlich}

\begin{abstract}
Der ErbB-Family-Blocker Afatinib bewährt sich auch bei mehrfach vorbehandelten NSCLC-Patienten. Selbst wenn eine Resistenz eintritt, lohnt sich die fortgesetzte Gabe in Kombination mit einem Chemotherapeutikum.
\end{abstract}

Patien atienten mit nichtkleinzelligem Lungenkarzinom (NSCLC) und aktivierenden $\operatorname{EGFR}($,epidermal growth factor receptor")-Mutationen sprechen in der Erstlinie gut auf EGFR-hemmende Tyrosinkinasehemmer (TKI) an. Praktisch alle Patienten werden nach einiger Zeit resistent. Experimentelle und klinische Befunde lassen jedoch vermuten, dass zum Zeitpunkt des resistenzbedingten Progresses das NSCLC-Gewebe sehr heterogen ist und immer noch TKI-empfindliche Zellen enthält. Eine weitere gegen EGFR gerichtete Behandlung, am besten unter Zugabe anderer Substanzen, könnte daher auch in dieser Situation noch sinnvoll sein. Ein solcher Ansatz wurde in der LUX-Lung-5-Studie mit Afatinib verfolgt. Dieser TKI hemmt neben dem EGFR-Signalweg u. a. HER2 (ErbB2). Aktiv ist er in der Erstlinie sowie nach Progress unter Gefitinib und/ oder Erlotinib. Die Studie wurde in 2 Teilen durchgeführt: Teil A umfasste Patienten nach Versagen einer Erstlinien-Chemotherapie sowie der nachfolgenden Erlotinib/Gefitinib-Behandlung. Die Patienten erhielten eine AfatinibMonotherapie zur Identifizierung derjenigen, deren Tumor noch auf eine ErbBBlockade ansprach. Der experimentelle B-Part umfasste 102 Patienten, die zunächst auf eine Afatinib-Gabe in der Dritt- oder Viertlinie angesprochen hatten, nach mindestens 12 Wochen aber refraktär geworden waren. Sie erhielten
Afatinib plus Paclitaxel oder eine Monochemotherapie. Primärer Endpunkt war das progressionsfreie Überleben (PFS).

Unter der Afatinib/Paclitaxel-Kombination war das PFS im Vergleich zur Monochemotherapie mit im Median 5,6 versus 2,8 Monaten signifikant länger (Hazard Ratio 0,60, p = 0,003). Auch die objektive Ansprechrate war deutlich höher ( 32,1 vs. $13,2 \%$; $p=0,005)$. Hinsichtlich des Gesamtüberlebens fanden sich dagegen keine Unterschiede. 48,5\% der Patienten entwickelten unter der Kombinationstherapie Nebenwirkungen vom Grad 3/4 im Vergleich zu 30\% unter der Monochemotherapie.

Fazit: Auch nach Progress der zunächst erfolgreichen alleinigen ErbB-Therapie zeigte sich ein Vorteil durch die Kombination mit Afatinib gegenüber einer Monochemotherapie. Barbara Kreutzkamp

Schuler $\mathrm{M}$ et al. Afatinib beyond progression in patients with non-small-cell lung cancer following chemotherapy, erlotinib/gefitinib and afatinib: phase III randomized LUX-Lung 5 trial. Ann Oncol. 2016;27(3):417-23.

\title{
Resezierbares NSCLC: Erlotinib wirksam bei aktivierenden EGFR-Mutationen
}

\begin{abstract}
Ist der Tyrosinkinasehemmer Erlotinib auch bei Patienten mit resezierbarem, EGFR-positivem nichtkleinzelligem Lungenkarzinom (NSCLC) wirksam? Der Nutzen in Erst- oder Zweitlinie wurde in der RADIANT-Studie überprüft.
\end{abstract}

$\mathrm{R}_{\mathrm{B}}^{\mathrm{A}}$ ADIANT (Randomized DoubleBlind Trial in Adjuvant NSCLC With Tarceva) ist eine doppelblinde Phase-IIIStudie mit NSCLC-Patienten im Stadium IB bis IIIA, deren Tumor EGFR („epidermal growth factor receptor") exprimierte. 2:1 randomisiert erhielten sie spätestens 3 Monate nach der Operation stratifiziert, u.a. entsprechend dem Stadium, der Histologie, der vorausgegangenen adjuvanten Chemotherapie oder dem Raucherstatus, 2 Jahre lang 1-mal täglich $150 \mathrm{mg}$ Erlotinib oder Placebo. Hinsichtlich des primären Endpunkts „krankheitsfreies Überleben“ (DFS) ließ sich bei den zwischen 2007 und 2010 randomisierten 973 Patienten kein signifikanter Unterschied feststellen (medi- an 50,0 vs. 48,2 Monate unter Placebo; Hazard Ratio [HR] 0,09, $95 \%$-Konfidenzintervall [95\%-KI] 0,74-1,10; $\mathrm{p}=0,324)$. In der präspezifizierten Subgruppe von Patienten mit EGFR-aktivierenden $\mathrm{Mu}$ tationen (EGFRm) ergab sich ein Trend zu einem besseren DFS (median 46,4 vs. 28,5 Monate; HR 0,61, $95 \%$-KI 0,38$0,98)$, doch erreichte auch diese Differenz aufgrund der Auswertungsmethodik keine Signifikanz. Die Daten zum Gesamtüberleben sind noch nicht reif.

Erwartungsgemäß war unter der Verummedikation Rash die häufigste Nebenwirkung, gefolgt von Diarrhö $(86,4$ und $52,2 \%$ vs. 32,1 und $15,7 \%$ unter Placebo). Im Nachhinein seien die Ergebnisse nicht überraschend, so die Forscher.
Auch in anderen Studien mit Tyrosinkinaseinhibitoren (TKI) beim fortgeschrittenen NSCLC zeigte sich in retrospektiven explorativen Biomarkeranalysen, dass eine alleinige EGFR-Expression auf Tumorzellen kein zuverlässiger Prädiktor für das Ansprechen auf TKI ist. Vielmehr scheinen aktivierende EGFR-Mutationen, wie beispielsweise L858R oder del19, eher geeignet, um das Ansprechen von NSCLC-Patienten auf EGFR-TKI vorherzusagen.

Fazit: Anders als bei Patienten mit fortgeschrittenem NSCLC, bei denen Erlotinib in der Zweitlinie wirksam war, ergab sich bei Patienten in frühen NSCLCStadien mit reseziertem Tumor kein statistisch signifikanter Unterschied im DFS im Vergleich zu Placebo. Prognostisch wertvoller für ein Ansprechen ist bei resektablen Patienten vermutlich der EGFRm-Status.

Barbara Kreutzkamp

Kelly K et al. Adjuvant erlotinib versus placebo in patients with stage IB-IIIA non-small-cell lung cancer (RADIANT): a randomized, double-blind, phase III trial. J Clin Oncol. 2015;33(34):4007-14. 